\title{
S100A9 promotes the proliferation and migration of cervical cancer cells by inducing epithelial-mesenchymal transition and activating the Wnt/ $\beta$-catenin pathway
}

\author{
HE ZHA ${ }^{1 *}$, XUERU LI ${ }^{2 *}$, HUI SUN ${ }^{3}$, LIANG DUAN ${ }^{4}$, SHIMEI YUAN ${ }^{4}$, HUAN LI $^{4}$, \\ ${\text { AIFANG } \mathrm{LI}^{4} \text {, YUE GU}}^{4}$, JIALI ZHAO ${ }^{4}$, JIAQING XIE ${ }^{4}$ and $\mathrm{LAN} \mathrm{ZHOU}^{4}$ \\ ${ }^{1}$ Department of Laboratory Medicine, The Third Affiliated Hospital of Zunyi Medical University, Zunyi, \\ Guizhou 563000; ${ }^{2}$ Sichuan Academy of Medical Sciences and Institute of Dermatology and Venereal Disease, \\ Sichuan Provincial People's Hospital, Chengdu, Sichuan 610031; ${ }^{3}$ Department of Laboratory Medicine, \\ The First Hospital of Xi'an, Xi'an, Shaanxi 710002; ${ }^{4}$ Key Laboratory of Clinical Diagnosis of Education Ministry, \\ College of Laboratory Medicine, Chongqing Medical University, Chongqing 400016, P.R. China
}

Received October 1, 2018; Accepted April 17, 2019

DOI: $10.3892 /$ ijo.2019.4793

\begin{abstract}
S100 calcium-binding protein A9 (S100A9), a member of the S100 protein family, is often upregulated in various cancers, including cervical cancer. Elevated S100A9 expression is thought to serve an important role in tumorigenesis; however, the exact role of S100A9 in the modulation of cervical cancer and the underlying molecular mechanism remain unknown. In the present study, we aimed to investigate the effects of S100A9 on the proliferation and migration of cervical cancer cells, as well as the molecular mechanisms underlying these effects. Our results demonstrated that endogenous expression of S100A9 in SiHa and CaSki cell lines was significantly higher than in the HeLa cell line. As expected, overexpression of S100A9 enhanced the proliferation and migration of cervical cancer cells. In addition, S100A9 overexpression induced epithelial-mesenchymal transition (EMT) as determined by reduced expression levels of the epithelial marker E-cadherin, whereas the expression levels of the mesenchymal marker vimentin were upregulated. Furthermore, it was reported that the effects of S100A9 in the modulation of cervical cancer cells were mediated through the Wnt/ $\beta$-catenin signaling pathway as $\beta$-catenin knockdown significantly suppressed the ability of S100A9 to enhance the proliferation and migration of cervical cancer cells. Collectively, these findings suggest that S100A9 promoted
\end{abstract}

Correspondence to: Dr Lan Zhou, Key Laboratory of Clinical Diagnosis of Education Ministry, College of Laboratory Medicine, Chongqing Medical University, 1 Yixueyuan Road, Chongqing 400016, P.R. China

E-mail: zhoulan@cqmu.edu.cn

${ }^{*}$ Contributed equally

Key words: S100A9, cervical cancer, proliferation, migration, EMT, Wnt/ $\beta$-catenin signaling pathway the proliferation and migration of cervical cancer cell lines. Furthermore, the underlying molecular mechanisms may be partially attributed to the induction of EMT and activation of the $\mathrm{Wnt} / \beta$-catenin signaling pathway.

\section{Introduction}

Cervical cancer ranks as the third most commonly diagnosed cancer and is the second leading cause of cancer-associated mortality in females worldwide (1). Despite improvements in screening and diagnostic techniques, regional or local relapse, and lymph node metastasis remain the most prevalent causes of cervical cancer-related mortality (2). Similarly, the motility and invasiveness of cancer cells serve a critical role in the mortality of patients with cervical cancer. Therefore, advances in understanding the mechanism of metastasis in cervical cancer may provide insight for developing effective approaches to prevent and treat cervical cancer.

S100A9, known as Calgranulin B or MRP-14, is a member of the low-molecular calcium binding S100 protein family. In our previous study, we demonstrated that exogenous S100A9 promoted the proliferation and invasion of HepG2 cells, which was partly mediated by activation of the MAPK signaling pathway (3) and that the underlying mechanism may be dependent on receptor of advanced glycation end products (RAGE)/MAPK signaling cascades (4). Interestingly, increasing evidence indicates that S100A9 is overexpressed in cervical cancer $(5,6)$ and could be a candidate marker for the early diagnosis of cervical cancer and a potential therapeutic target for therapy(5). few studies have investigated the precise role of S100A9 in the tumorigenesis and development of cervical cancer, and the underlying potential molecular mechanism.

Epithelial-mesenchymal transition (EMT) is a vital process associated with the pathogenesis of numerous cancers, including cervical cancer. This transition is characterized by a process in which epithelial cells lose their polarity enabling them to assume a mesenchymal cell phenotype, which imparts cancer 
cells several properties, such as tumor invasion, carcinoma metastasis and chemotherapy resistance (7). A previous study demonstrated that cellular RAGE binding to S100A8/A9 can cause a notable increase and accumulation of endogenous Snail in the nucleus of breast cancer cells; thus, EMT and cancer metastasis are enhanced via the nuclear factor $(N F)-\kappa B$ signaling module (8). Additionally, the effects of transforming growth factor $\beta 1$ on EMT were antagonized by S100A9 via the formation of heterodimers between them in pancreatic cancer; however, the effects of S100A9 on EMT in cervical cancer require further investigation.

Recent studies reported that cytoplasmic/nuclear expression of $\beta$-catenin was associated with the malignant phenotype and the pathogenesis of cervical cancer $(9,10)$. In addition, it was indicated that the $\mathrm{Wnt} / \beta$-catenin pathway and EMT may act synergistically in the progression of cervical cancer (11). Tumor cells exhibiting $\beta$-catenin accumulation in the nucleus appear to promote EMT, which involves the loss of epithelial markers and the expression of mesenchymal markers (12). Members of the S100 protein family, including S100A6, S100B, and S100P were reported to negatively regulate $\beta$-catenin degradation. Our consensus finding is that recombinant S100A8 and S100A9 proteins could promote the accumulation of $\beta$-catenin, and upregulate mRNA expression of the target genes of the $\mathrm{Wnt} / \beta$-catenin signaling pathway in colorectal carcinoma (13). However, whether S100A9 regulates the $\mathrm{Wnt} / \beta$-catenin pathway, thereby exerting its effects on the progression of cervical cancer remains unknown.

In the present study, the functional role of S100A9 in the Wnt/ $\beta$-catenin signaling pathway and EMT was investigated to elucidate how S100A9 modulates the progression of cervical cancer. We reported that S100A9 promoted the proliferation, migration, and EMT of cervical cancer cells, and the activation of the $\mathrm{Wnt} / \beta$-catenin signaling pathway may be involved in the modulation of these processes. These findings provide novel insight to the importance of S100A9 in the progression of cervical cancer and a basis for the development of targeted therapeutic strategies for treating cervical cancer.

\section{Materials and methods}

Cell culture. The human embryonic kidney cell line 293 and the human cervical cancer cell lines SiHa, CaSki, and HeLa were obtained from the American Type Culture Collection. All cells were maintained in Dulbecco's modified Eagle's medium (DMEM) supplemented with fetal bovine serum (FBS; 10\%; both obtained from Gibco; Thermo Fisher Scientific, Inc.), $100 \mathrm{U} / \mathrm{ml}$ penicillin and $100 \mu \mathrm{g} / \mathrm{ml}$ streptomycin (both obtained from HyClone; GE Healthcare) at $37^{\circ} \mathrm{C}$ in a humidified atmosphere of $5 \% \mathrm{CO}_{2} .293$ cells were used for adenovirus amplification.

Reagents and antibodies. Adenovirus expressing S100A9 and green fluorescent protein (AdS100A9), adenovirus expressing small interfering (si)RNA targeting $\beta$-catenin and red fluorescent protein (Adsi $\beta$-catenin), control adenovirus expressing green fluorescent protein (AdGFP) and red fluorescent protein (AdRFP), and pGST-moluc and pGST-moluc-hS100A9 plasmids were kindly donated by Professor T.C. He (The University of Chicago Medical Center, Chicago, IL, USA). Competent bacteria E. coli (BL21) were saved in our laboratory. Adenoviruses expressing siRNA targeting S100A9 and red fluorescent protein (AdsiS100A9), and control adenoviruses expressing red fluorescent protein (AdsiControl) were constructed in house. The kit used for semi-quantitative PCR was purchased from Takara Bio, Inc. Antibodies, including mouse anti- $\beta$-actin, anti-vimentin and anti- $\beta$-catenin were purchased from Santa Cruz Biotechnology, Inc. (cat. nos. sc-47778, sc-66001 and sc-59737). Rabbit anti-S100A9 antibody was purchased from Abcam (cat. no. ab92507). Rabbit anti-E-cadherin antibody was purchased from ImmunoWay (cat. no. YM3353, Plano). Rabbit anti-histone H3 antibody was purchased from Abmart (cat. no. P30266). Secondary antibody reagents, such as goat anti-mouse IgG serum and goat anti-rabbit IgG serum were obtained from Beijing Zhongshan Golden Bridge Biotechnology (cat. no. 2305 and no. 2301). Western blot reagents and radioimmunoprecipitation assay (RIPA) buffer were purchased from Beyotime Institute of Biotechnology. Phosphatase and protease inhibitors were purchased from Roche Diagnostics GmbH. Polyvinylidene difluoride (PVDF) membranes and an enhanced chemiluminescence (ECL) kit were purchased from EMD Millipore.

Adenovirus infection. HeLa cells were infected with AdS100A9 and AdGFP, whereas SiHa cells were infected with AdsiS100A9 and AdsiControl. After 8-12 h of incubation, the medium was replaced with complete medium containing FBS followed by continued cell culture for subsequent experiments. The cells were maintained at $37^{\circ} \mathrm{C}$ in a humidified atmosphere of $5 \% \mathrm{CO}_{2}$.

Recombinant protein preparation. The pGST-moluc and pGST-moluc-hS100A9 plasmids used in the present study has been described previously (4). In brief, pGST-moluc and pGST-moluc-hS100A9 was transfected into $E$. coli (BL21) by calcium chloride-mediated transformation. Isopropylthio- $\beta$-D-galactoside was used to induce the expression of GST and GST-hS100A9 proteins. The bacteria were then collected and sonicated on ice at $4^{\circ} \mathrm{C}$. The supernatants were incubated with glutathione-sepharose $4 \mathrm{~B}$ beads, GST and GST-hS100A9 proteins on the beads were eluted by elution buffer with reduced glutathione on ice. Finally the GST and GST-hS100A9 proteins were filtered and stored at $-80^{\circ} \mathrm{C}$. Cells were treated with $20 \mu \mathrm{g} / \mathrm{ml}$ of recombinant proteins for $48 \mathrm{~h}$ at $37^{\circ} \mathrm{C}$.

RNA extraction and semi-quantitative polymerase chain reaction $(P C R)$ analysis. Total RNA was extracted from cells using TRIzol ${ }^{\circledR}$ reagent (Invitrogen; Thermo Fisher Scientific, Inc.), and cDNA samples were synthesized using random primers and a Reverse Transcriptase PCR kit (Takara Bio, Inc.) according to the manufacturer's protocols. cDNA was used as a template for semi-quantitative PCR. The thermocycling conditions were as follows: $94^{\circ} \mathrm{C}$ for $5 \mathrm{~min}, 94^{\circ} \mathrm{C}$ for $30 \mathrm{sec}$, $68^{\circ} \mathrm{C}$ for $30 \mathrm{sec}$ and $72^{\circ} \mathrm{C}$ for 12 cycles with a decrease in $1^{\circ} \mathrm{C} /$ cycle; then, $94^{\circ} \mathrm{C}$ for $30 \mathrm{sec}, 55^{\circ} \mathrm{C}$ for $30 \mathrm{sec}$, and $72^{\circ} \mathrm{C}$ for $30 \mathrm{sec}$ for $18-27$ cycles depending on the abundance of the target genes. The PCR products were identified by 
Table I. Primers employed in the present study.

Gene

\section{S100A9}

c-Myc

Snail

Twist

GAPDH
Primer sequences

Product size (bp)

Forward: 5'-ACCCAGACACCCTGAACC-3'

Reverse: 5'-AGCATGATGAACTCCTCGA-3'

Forward: 5'-TACCCTCTCAACGACAGCAG-3'

Reverse: 5'-TCTTGACATTCTCCTCGGTG-3'

Forward: 5'-ACCCCACATCCTTCTCACTG-3'

Reverse: 5'-TACAAAAACCCACGCAGACA-3'

Forward: 5'-TCTTACGAGGAGCTGCAGAC-3'

Reverse: 5'-TATCCAGCTCCAGAGTCTCT-3'

406

Forward: 5'-CAGCGACACCCACTCCTC-3'

Reverse: 5'-TGAGGTCCACCACCCTGT-3' electrophoresis using $1.5 \%$ agarose gels. GAPDH was used as an internal reference control. The results were recorded using Gel Doc 1000 imaging system and Quantity One version 4.5.0 software (Bio-Rad Laboratories, Inc.). The primers used in this study are shown in Table I.

Western blot analysis. Total cellular protein of cervical cancer cells were prepared using RIPA buffer containing phosphatase and protease inhibitors. Nuclear and cytoplasmic proteins were extracted using Nuclear-Cytosol Extraction Kit (cat. no. KGP1100, Nanjing KeyGen Biotech Co., Ltd.) according to the manufacturer's instructions. Protein extracts were separated by 6-15\% SDS-PAGE and transferred to PVDF membranes. The membranes were blocked with $5 \%$ bovine serum albumin at $37^{\circ} \mathrm{C}$ for $2 \mathrm{~h}$, washed with Tris-buffered saline with Tween-20 (TBST) and incubated with primary antibodies against S100A9, vimentin, E-cadherin, $\beta$-catenin, histone $\mathrm{H} 3$, and $\beta$-actin separately (1:1,000 dilution) at $4^{\circ} \mathrm{C}$ overnight. After washing with TBST, the membranes were incubated with the a secondary antibody conjugated with horseradish peroxidase (1:5,000 dilution) for $1 \mathrm{~h}$ at $37^{\circ} \mathrm{C}$. Finally, the blots were washed with TBST, and then visualized using the Bio-Rad Gel Doc 1000 imaging system and analyzed by Quantity One Version 4.5.0.

Cell proliferation assay. Cell proliferation was analyzed via an MTT assay (Promega Corporation). A total of $3.5 \times 10^{3} \mathrm{HeLa}$ cells or $4.5 \times 10^{3} \mathrm{SiHa}$ cells were seeded in 96-well flat-bottomed microplates. After infection with or without adenoviruses in DMEM containing 1\% FBS for 24,48 and $72 \mathrm{~h}, 10 \mu \mathrm{l}$ of MTT reagent was added to each well and incubated at $37^{\circ} \mathrm{C}$ for $4 \mathrm{~h}$. Dimethyl sulfoxide $(100 \mu \mathrm{l})$ was added to dissolve the formazan crystals for $10 \mathrm{~min}$ at room temperature. Finally, the absorbance was measured at $490 \mathrm{~nm}$ using an automatic enzyme immunoassay analyzer (Bio-Rad Laboratories, Inc.). The experiments were performed five times in three independent experimental trials.

Wound healing assay. Cervical cancer cells were seeded in 6 -well plates and incubated at $37^{\circ} \mathrm{C}$ until the cells attained $90 \%$ confluence. The cells were then infected with adenoviruses. Cell monolayers were carefully wounded by scratching with a sterile $10-\mu l$ pipette tip. The cells were then washed twice with cold PBS and incubated in serum-free DMEM at $37^{\circ} \mathrm{C}$. Wound areas were observed and imaged at $\mathrm{x} 100$ magnification at 0,24 and $36 \mathrm{~h}$ with an inverted fluorescent microscope (Nikon eclipse 80i; Nikon Corporation). Overall, the experiments were repeated three times.

Transwell migration assay. A chamber of non-type I-collagencoated 24-well Transwell cell culture inserts (EMD Millipore) was used for the Transwell migration assay. Briefly, after infection with or without adenoviruses and recombination proteins for various durations, the cells were trypsinized, washed, and suspended in $400 \mu \mathrm{l}$ of serum-free DMEM and finally seeded in the upper chamber (HeLa, $4 \times 10^{4}$ cells per well; SiHa, $6 \times 10^{4}$ cells per well). The lower chamber was coated with $700 \mu 1$ of DMEM containing $20 \%$ FBS as a chemoattractant. After $24 \mathrm{~h}$, cells were fixed with methanol for $20 \mathrm{~min}$ and stained with $0.05 \%$ crystal violet for $30 \mathrm{~min}$ at room temperature. Cells on the upper surface of the insert membrane were removed with cotton swabs. Finally, the cells were counted using an inverted microscope (magnification, x100) in five randomly selected fields for each well. The experiment was repeated three times.

Immunofluorescence. The cells were seeded on sterile glass coverslips in 24-well culture plates. After treatment with or without AdS100A9, cells were washed with PBS, fixed with $4 \%$ paraformaldehyde for $30 \mathrm{~min}$ and permeabilized with $0.3 \%$ Triton $\mathrm{X}-100$ at $37^{\circ} \mathrm{C}$ for $30 \mathrm{~min}$. After blocking with goat serum at $37^{\circ} \mathrm{C}$ for $30 \mathrm{~min}$, the cells were incubated with antibodies against $\mathrm{E}$-cadherin, $\mathrm{N}$-cadherin (1:100 dilution in PBS) at $4^{\circ} \mathrm{C}$ overnight. Subsequently, slides were washed with PBS and incubated with fluorescein isothiocyanate-conjugated secondary antibodies (Beyotime Institute of Biotechnology) for $1 \mathrm{~h}$ at room temperature in the dark. Then, the cells were washed with PBS, counter stained with DAPI (Beyotime Institute of Biotechnology) for $5 \mathrm{~min}$ at room temperature, washed with PBS again and then the coverslips were mounted using antifade mounting medium. The fluorescence of the various groups was visualized imaged with a Nikon eclipse 80i inverted fluorescent microscope (Nikon Corporation). 
A
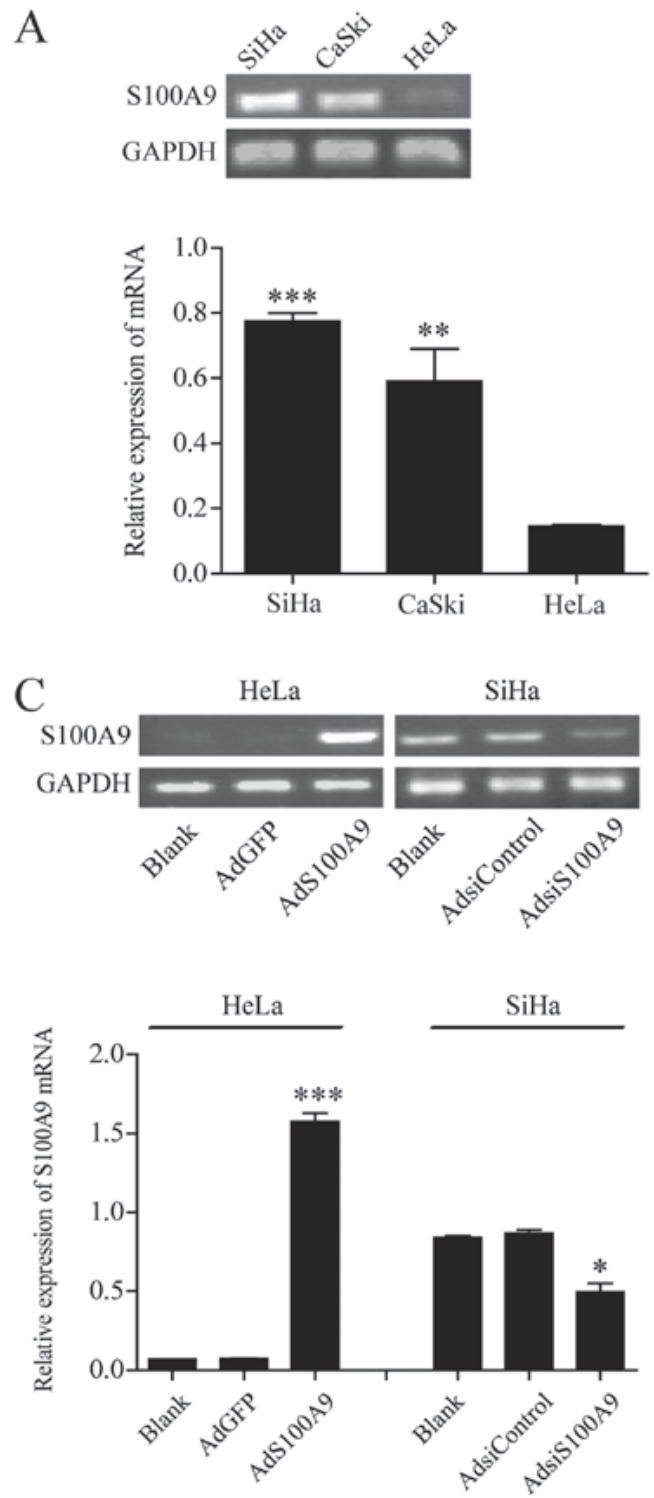

$\mathrm{B}$
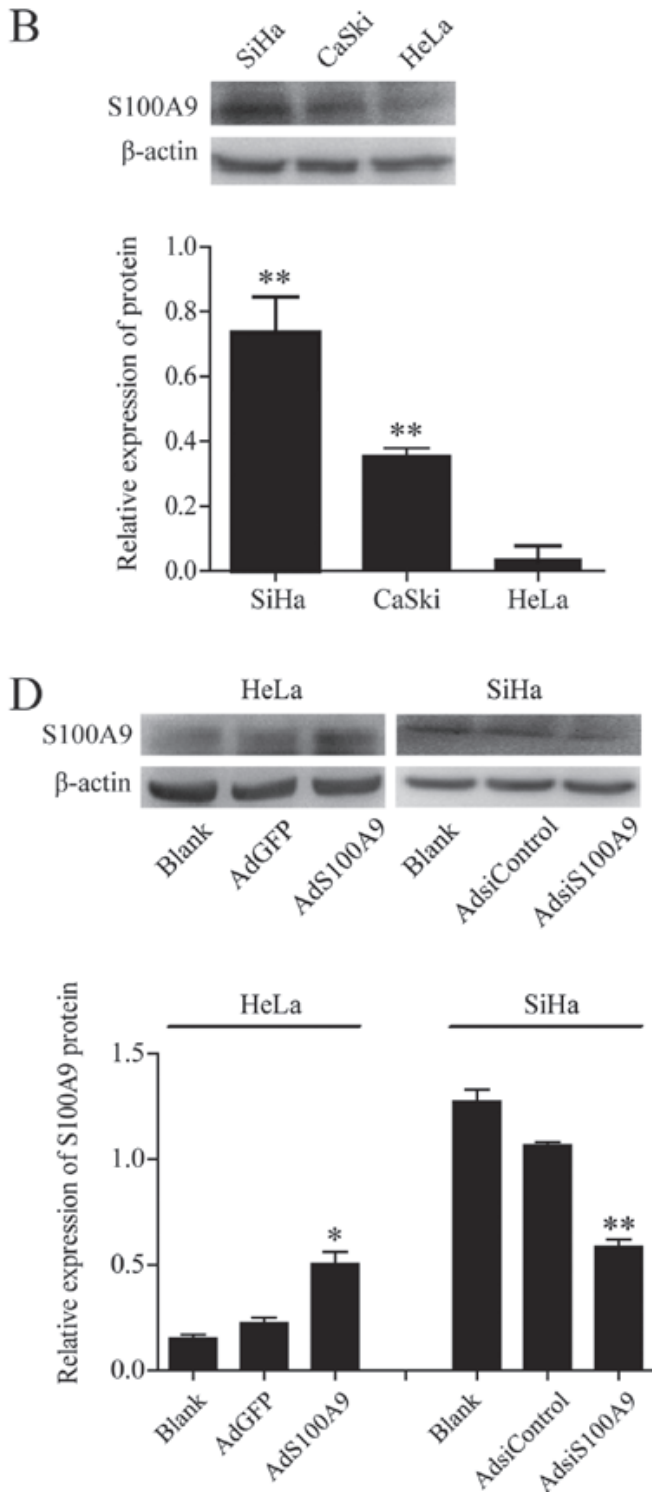

Figure 1. S100A9 is successfully overexpressed in HeLa cells and downregulated in SiHa cells via adenoviral infection. (A) mRNA expression of endogenous S100A9 in SiHa, CaSki and HeLa cell lines was detected by semi-quantitative PCR analysis. ${ }^{* * *} \mathrm{P} \leq 0.001, \mathrm{HeLa}$ vs. SiHa and ${ }^{* *} \mathrm{P} \leq 0.01, \mathrm{HeLa}$ vs. CaSki. (B) Protein expression of endogenous S100A9 in SiHa, CaSki and HeLa cell lines was detected by western blot analysis. ${ }^{* *} \mathrm{P} \leq 0.01$, HeLa vs. SiHa and CaSki. HeLa cells were infected with AdGFP and AdS100A9 for $48 \mathrm{~h}$, whereas SiHa cells were infected with AdsiControl and AdsiS100A9 for 48 h. The mRNA and protein expression of S100A9 in cervical carcinoma cell lines was detected by (C) semi-quantitative PCR and (D) western blot analysis. Data are presented as the mean \pm standard deviation of three independent experiments. ${ }^{* * *} \mathrm{P} \leq 0.001,{ }^{* * *} \mathrm{P} \leq 0.01$ and ${ }^{*} \mathrm{P} \leq 0.05$, AdS100A9 vs. AdGFP and AdsiControl. No significant difference between the AdGFP/AdsiControl and Blank groups of HeLa and $\mathrm{SiHa}$ cell lines were observed $(\mathrm{P}>0.05)$. Ad, adenovirus; Adsi, adenovirus containing small interfering RNA; GFP, green fluorescent protein; PCR, polymerase chain reaction.

Statistical analysis. All data are presented as the mean \pm standard deviation. Differences were analyzed using one-way ANOVA followed by a Tukey's multiple comparison test. All statistical analyses were performed using SPSS statistical package 17.0 (SPSS, Inc., Chicago, IL, USA) and GraphPad Prism 5 (GraphPad Software, Inc.). P $\leq 0.05$ was considered to indicate a statistically significant difference.

\section{Results}

Expression of endogenous S100A9 in cervical cancer cells. We first evaluated the endogenous expression of S100A9 in $\mathrm{SiHa}, \mathrm{CaSki}$, and HeLa cell lines using semi-quantitative PCR and western blot analysis. As shown in Fig. 1A, the mRNA expression levels of S100A9 in HeLa cells were significantly reduced than in $\mathrm{SiHa}$ and CaSki cells $(\mathrm{P} \leq 0.001$ and $\mathrm{P} \leq 0.01)$. These results were confirmed by western blot analysis $(\mathrm{P} \leq 0.01$; Fig. 1B). Subsequently, semi-quantitative PCR and western blot analysis (Fig. 1C and D) were performed to detect the expression of S100A9 in HeLa and SiHa cells infected with AdS100A9 and AdsiS100A9 for $48 \mathrm{~h}$, respectively. The expression of S100A9 observed in the AdS100A9 group was significantly increased compared with the AdGFP and Blank groups in HeLa cells $(\mathrm{P} \leq 0.001$ and $\mathrm{P} \leq 0.05)$, whereas a significant decrease in S100A9 expression was detected in the AdsiS100A9 group compared with AdsiControl and Blank groups in SiHa cells $(\mathrm{P} \leq 0.05$ and $\mathrm{P} \leq 0.01)$. These results indicated that S100A9 was successfully overexpressed in 

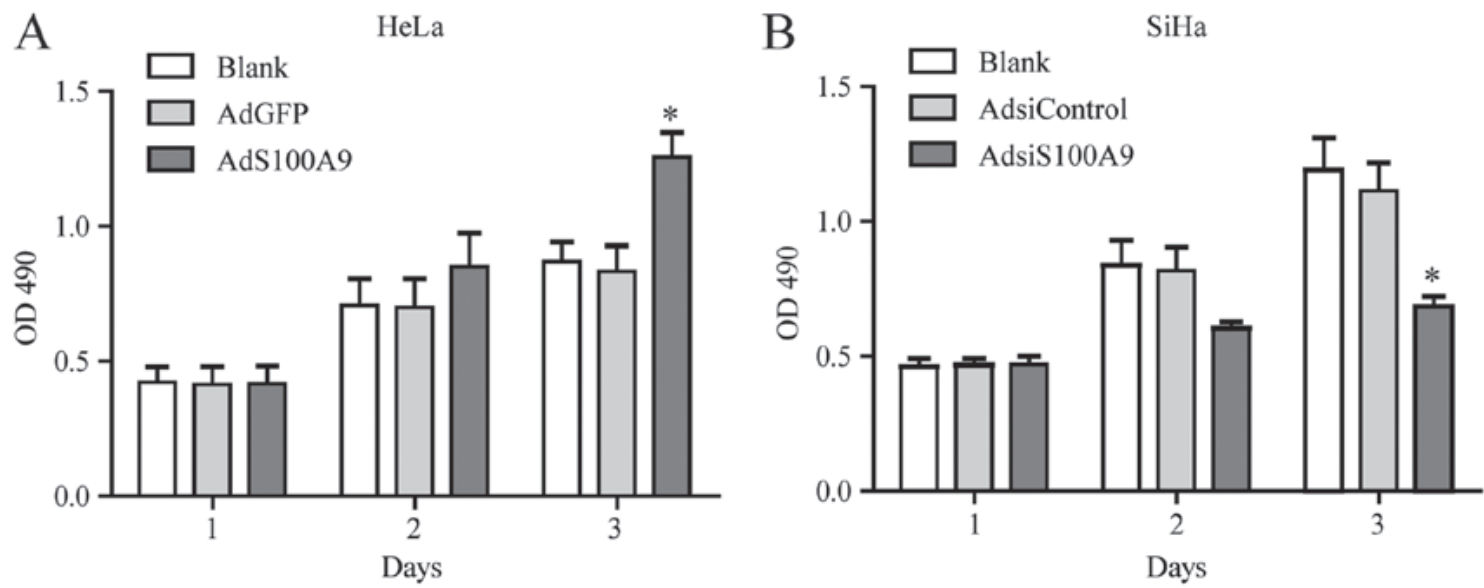

Figure 2. S100A9 upregulates the proliferation of cervical cancer cells. The proliferation of cervical cancer cells was determined by an MTT assay. (A) HeLa cells were infected with AdGFP or AdS100A9 for 3 days. (B) SiHa cells were infected with AdsiControl or AdsiS100A9 for 3 days. Data are presented as the mean \pm standard deviation of three independent experiments. "P $\leq 0.05$, AdS100A9 vs. GFP and AdsiControl. Ad, adenovirus; Adsi, adenovirus containing small interfering RNA; GFP, green fluorescent protein.

HeLa cells and downregulated in SiHa cells via infection with AdS100A9 and AdsiS100A9, respectively.

S100A9 promotes the proliferation of cervical cancer cells. To investigate the effects of S100A9 on the proliferation of cervical cancer cells, we treated the cells with AdS100A9 and AdsiS100A9 for 3 days and examined by an MTT assay (Fig. 2). Compared with the control groups, the proliferation of HeLa cells was significantly enhanced in the S100A9-overexpressing group on day 3 (P $\leq 0.05 ;$ Fig. 2A). On the contrary, the proliferation of $\mathrm{SiHa}$ cells was significantly inhibited in the S100A9-knockdown group compared with the control ( $\mathrm{P} \leq 0.05$; Fig. $2 \mathrm{~B})$. These results demonstrated that overexpressed S100A9 could promote the proliferative potential of cervical cancer cells.

S100A9 promotes the migration of cervical cancer cells. We further studied the role of S100A9 in the migration of cervical cancer cells by wound healing and Transwell migration assays. The results revealed that the wound closure rate of S100A9-overexpressing HeLa cells was notably increased than the control group, whereas that of SiHa cells was impaired following knockdown of S100A9 (Fig. 3A). Similar results were observed in the Transwell migration assay. The number of HeLa cells migrated across the membrane in AdS100A9 group was significantly higher than that in the AdGFP group ( $\mathrm{P} \leq 0.05$; Fig. 3B). These results are consistent with the wound healing ability of SiHa cells. The number of S100A9-depleted $\mathrm{SiHa}$ cells that migrated across the membrane was significantly lower compared with the control $(\mathrm{P} \leq 0.01)$. Collectively, these results suggested that upregulated S100A9 expression may facilitate the migration of cervical cancer cells.

S100A9 induces EMT in cervical cancer cells. Emerging evidences support that EMT has a crucial role in the metastasis of primary tumors. To evaluate whether S100A9 promoted EMT in cervical cancer cells, western blot and immunofluorescence analyses were conducted to detect the levels of E-cadherin and vimentin. As presented in Fig. 4A, overexpression of S100A9 in HeLa cells significantly decreased the expression of E-cadherin $(\mathrm{P} \leq 0.05)$, and increased that of of vimentin $(\mathrm{P} \leq 0.01)$. On the contrary, knockdown of S100A9 in $\mathrm{SiHa}$ cells significantly decreased the expression of vimentin $(\mathrm{P} \leq 0.01)$, and increased that of E-cadherin $(\mathrm{P} \leq 0.05)$. These results were further confirmed by immunofluorescence assays (Fig. 4B). These findings suggested that S100A9 is involved in the regulation of EMT-related protein expression, and may act as a predictive factor and therapeutic target for preventing metastasis in cervical cancer.

S100A9 promotes the activation of the Wnt/ $\beta$-catenin signaling pathway in cervical cancer cells. It is well known that aberrant activation of the Wnt/ $\beta$-catenin pathway serves a critical role in the development of cervical cancer. To investigate whether S100A9 was involved in the regulation of Wnt/ $\beta$-catenin pathway in cervical cancer cells, western blot and semi-quantitative PCR analyses were performed to study the effects of S100A9 on the expression of $\beta$-catenin protein, and the transcriptional levels of c-Myc, Snail, and twist-related protein-1 (Twist), which are the classical target genes of Wnt/ $\beta$-catenin pathway. As shown in Fig. 5A, after infecting HeLa cells with AdS100A9, overexpression of S100A9 significantly enhanced the accumulation of total $\beta$-catenin and nuclear $\beta$-catenin $(\mathrm{P} \leq 0.01)$, but knockdown of S100A9 via the infection of SiHa cells with AdsiS100A9 decreased the accumulation of total $\beta$-catenin and nuclear $\beta$-catenin compared with the control groups $(\mathrm{P} \leq 0.001$ and $\mathrm{P} \leq 0.05$ ). Additionally, overexpression of S100A9 caused by infecting HeLa cells with AdS100A9 significantly promoted the transcription of c-Myc $(\mathrm{P} \leq 0.05)$, Snail $(\mathrm{P} \leq 0.01)$, and Twist $(\mathrm{P} \leq 0.05)$ compared with the control. Conversely, the mRNA levels of the aforementioned target genes of the Wnt/ $\beta$-catenin signaling pathway were significantly reduced when $\mathrm{SiHa}$ cells were infected with AdsiS100A9 compared with the control $(\mathrm{P} \leq 0.01, \mathrm{P} \leq 0.01$ and $\mathrm{P} \leq 0.001$, respectively; Fig. 5B). These results demonstrated that S100A9-induced upregulation of c-Myc, Snail, and Twist expression in cervical cancer cell lines may be associated with the accumulation of total $\beta$-catenin and nuclear $\beta$-catenin. 
A

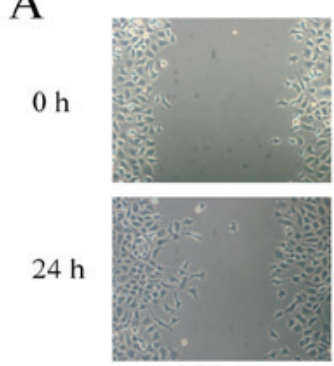

Blank
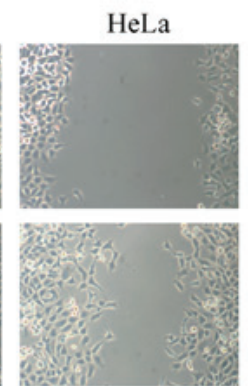

AdGFP
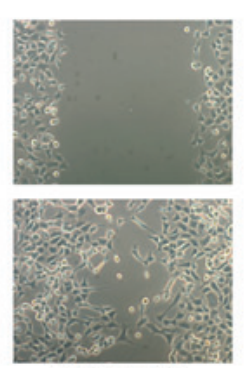

AdS100A9
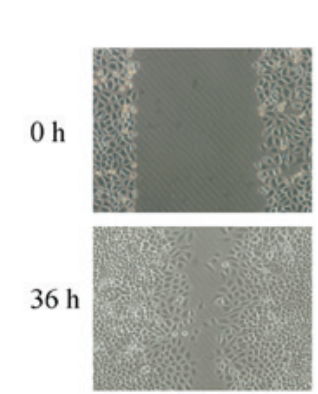

Blank
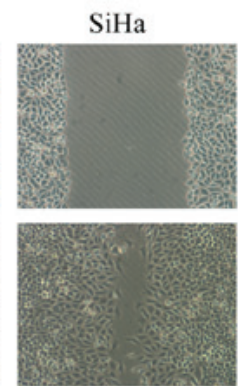

AdsiControl
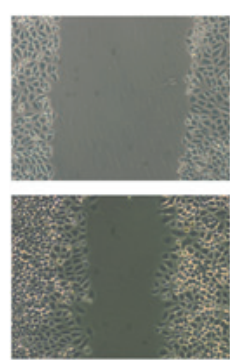

AdsiS100A9

B

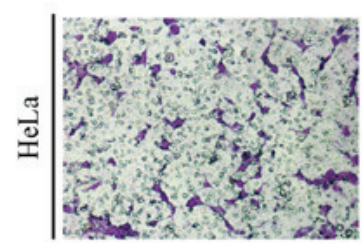

Blank

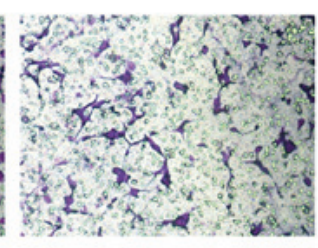

AdGFP

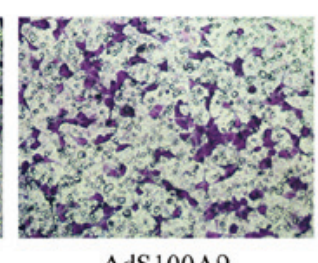

AdS100A9

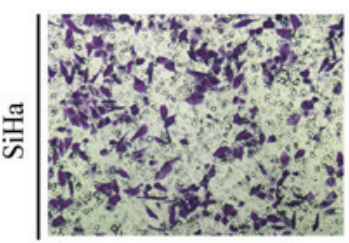

Blank

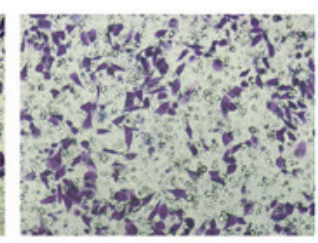

AdsiControl

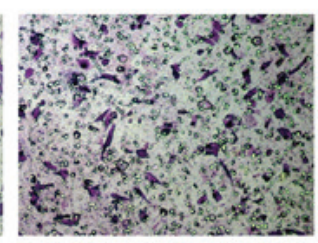

AdsiS100A9
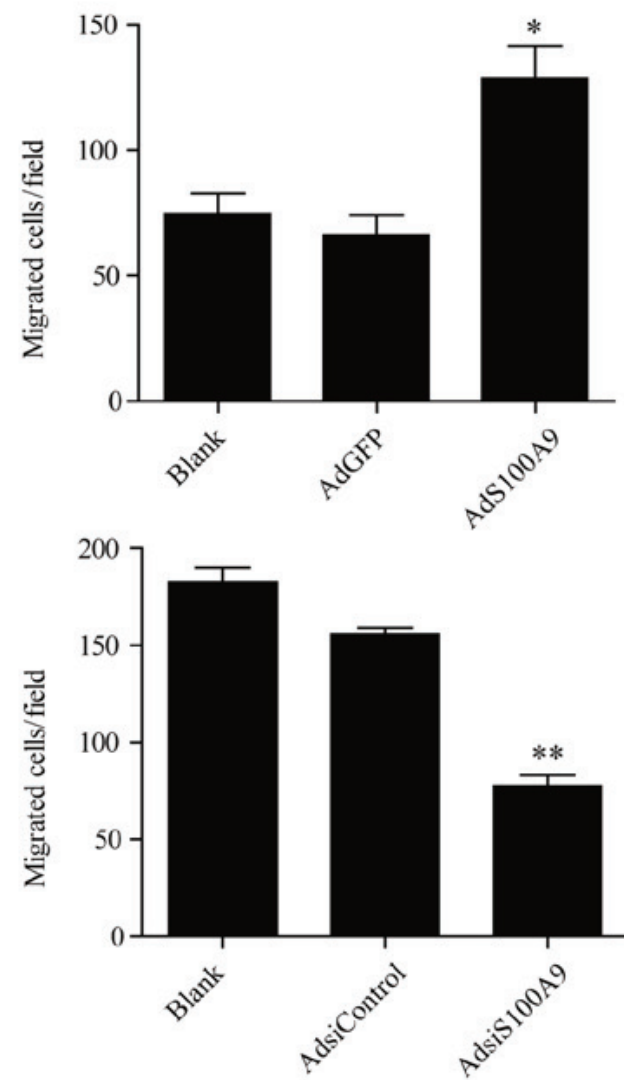

Figure 3. S100A9 promotes the migration of cervical cancer cells. (A) HeLa and SiHa cells were infected with AdS100A9 and AdsiS100A9, respectively, and the migration ability was determined by wound healing assay. The wound width was measured. (B) Transwell migration assay for analyzing the migration of HeLa and SiHa cells that were infected with AdS100A9 or AdsiS100A9 for $48 \mathrm{~h}$. Representative images of transmembrane cells are shown. Data are presented as the mean \pm standard deviation of three individual measurements. ${ }^{*} \mathrm{P} \leq 0.05$, AdS100A9 vs. AdGFP group; ${ }^{* *}$ P $\leq 0.01$, AdsiS100A9 vs. AdsiControl group. Ad, adenovirus; Adsi, adenovirus containing small interfering RNA; GFP, green fluorescent protein.

S100A9-induced promotion of proliferation, migration and EMT in cervical cancer cells via activation of the Wnt/ $\beta$-catenin pathway. To determine whether Wnt/ $\beta$-catenin pathway is involved in S100A9-induced promotion of proliferation, migration and EMT in cervical cancer cells, adenovirus Adsi $\beta$-catenin was used as the antagonist to impair the activation of the $\mathrm{Wnt} / \beta$-catenin pathway. As presented in Fig. 6A, the effects of S100A9 on the proliferation of HeLa cells was investigated by an MTT assay. After the treatment of cells with GST-hS100A9 at 10 and $20 \mu \mathrm{g} / \mathrm{ml}$ for $72 \mathrm{~h}$, the proliferation of HeLa cells was significantly increased than that of the control groups $(\mathrm{P} \leq 0.05)$. Additionally, the results further showed a more notable effect in response to high concentration recombinant protein; thus, we selected $20 \mu \mathrm{g} / \mathrm{ml}$ of recombinant protein for the following experiments. As shown in Fig. 6B, $\beta$-catenin expression was significantly downregulated by adenovirus Adsi $\beta$-catenin in HeLa cells compared with the control $(\mathrm{P} \leq 0.05)$. After treatment with GST-hS100A9 and adenovirus Adsi $\beta$-catenin, the mRNA levels of Snail, and Twist were significantly reduced in HeLa cells compared with the control groups $(\mathrm{P} \leq 0.05$ and $\mathrm{P} \leq 0.01$; Fig. 6C). In addition, treatment with Adsi $\beta$-catenin significantly inhibited the proliferation and migration of HeLa cells induced by GST-hS100A9 $(\mathrm{P} \leq 0.05$ and $\mathrm{P} \leq 0.01$; Fig. 6D and E). Furthermore, the expression of EMT markers was examined in HeLa cells after treatment with and without AdRFP, Adsi $\beta$-catenin, GST and GST-hS100A9. We observed that downregulation of $\beta$-catenin significantly suppressed the effects of S100A9 on the expression of E-cadherin and vimentin compared with the control groups $(\mathrm{P} \leq 0.05$ and 
A

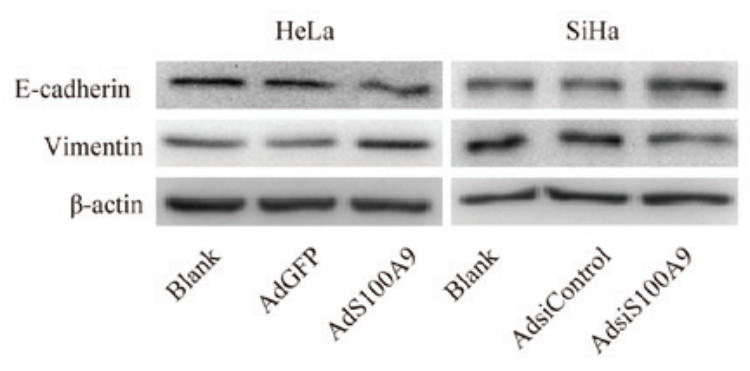

B
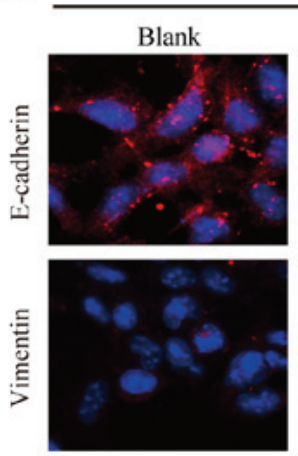

HeLa
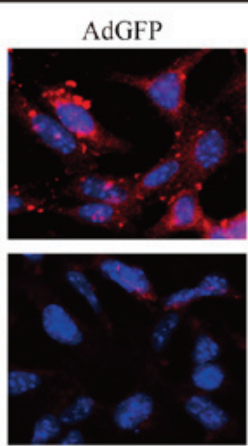

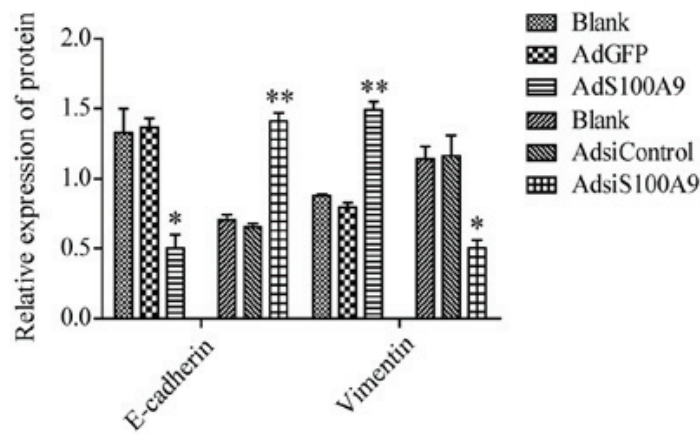

$\mathrm{SiHa}$
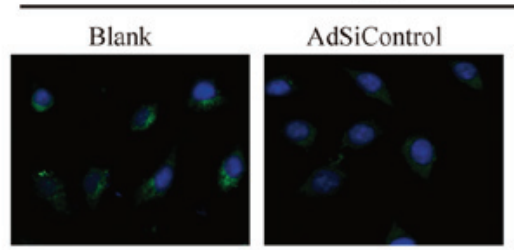

AdsiS100A9
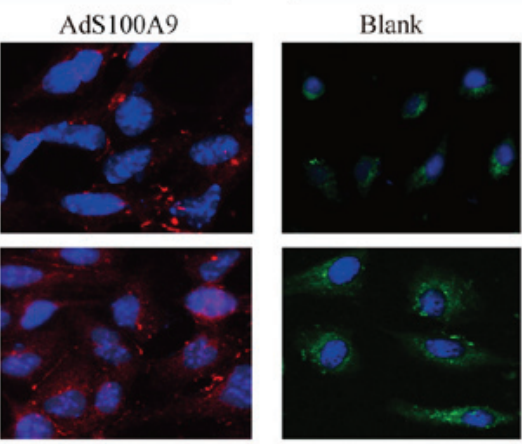
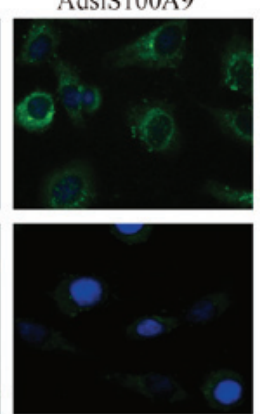

Figure 4. S100A9 promotes EMT in cervical cancer cells. After HeLa and SiHa cells were infected with AdS100A9 and AdsiS100A9, respectively, (A) the expression of EMT markers was detected by western blot analysis. ${ }^{*} \mathrm{P} \leq 0.05,{ }^{* *} \mathrm{P} \leq 0.01$, AdS100A9 vs. AdGFP and AdsiControl. Data are presented as the mean \pm standard deviation of three individual measurements. (B) The levels of EMT markers were detected by immunofluorescence assays. The representative images are presented. Ad, adenovirus; Adsi, adenovirus containing small interfering RNA; EMT, epithelial-mesenchymal transition; GFP, green fluorescent protein.

A

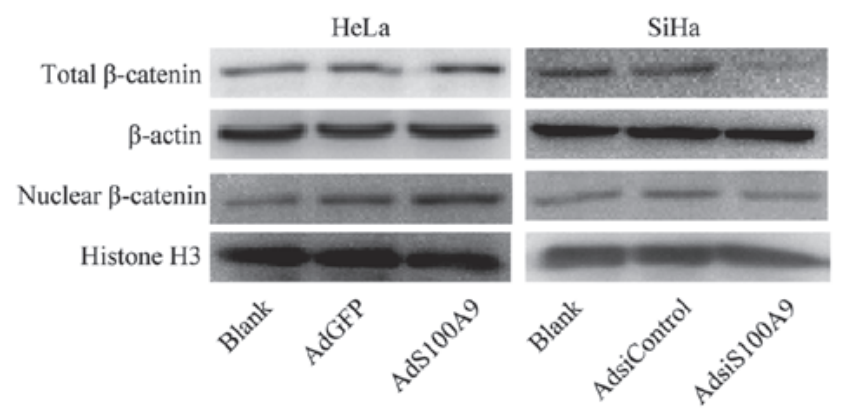

B

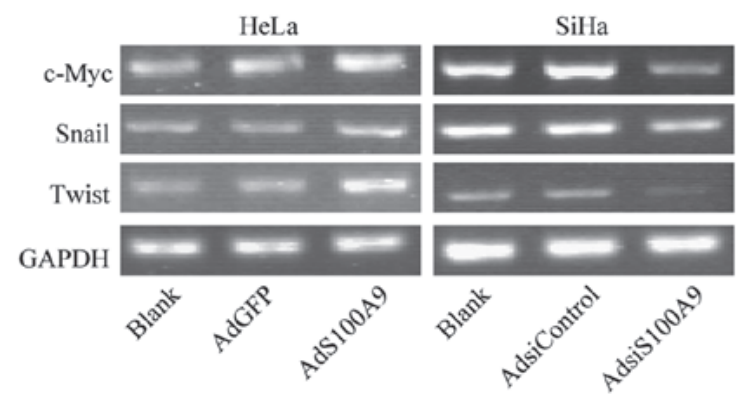

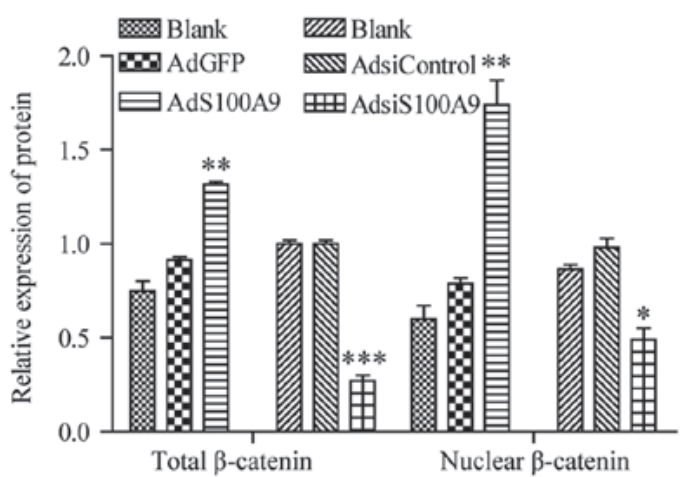

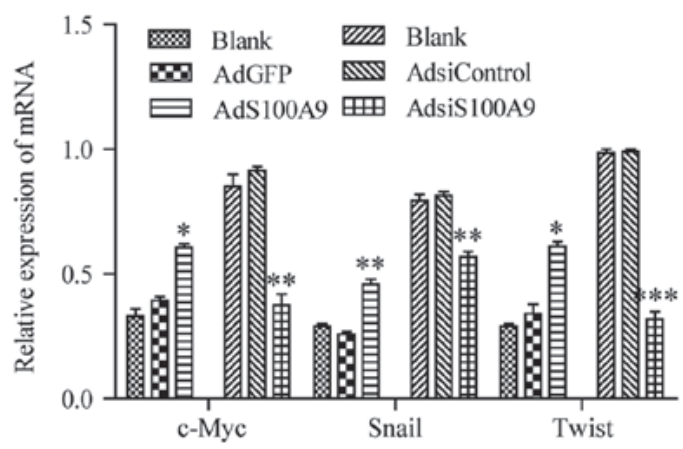

Figure 5. S100A9 activates the Wnt/ $\beta$-catenin signaling pathway in cervical cancer cells. (A) After HeLa and SiHa cells were infected with AdS100A9 or AdsiS100A9, the accumulation of total and nuclear $\beta$-catenin was measured by western blot analysis. $\beta$-actin and histone were used as internal reference controls. ${ }^{* *} \mathrm{P} \leq 0.01$, AdS100A9 vs. AdGFP; ${ }^{* * *} \mathrm{P} \leq 0.001,{ }^{*} \mathrm{P} \leq 0.05$, AdsiS100A9 vs. AdsiControl. (B) After infecting HeLa and SiHa cells with AdS100A9 or AdsiS100A9, the mRNA expression of c-Myc, Snail, and Twist was detected using semi-quantitative polymerase chain reaction. GAPDH was used as an internal reference control. ${ }^{*} \mathrm{P} \leq 0.05,{ }^{* *} \mathrm{P} \leq 0.01,{ }^{* * *} \mathrm{P} \leq 0.001$, AdS100A9 vs. AdGFP and AdsiControl. Data are presented as mean \pm standard deviation of three individual measurements. Ad, adenovirus; Adsi, adenovirus containing small interfering RNA; Twist, twist-related protein-1. 


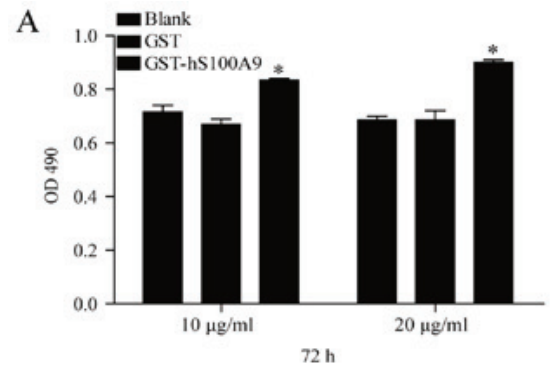

C
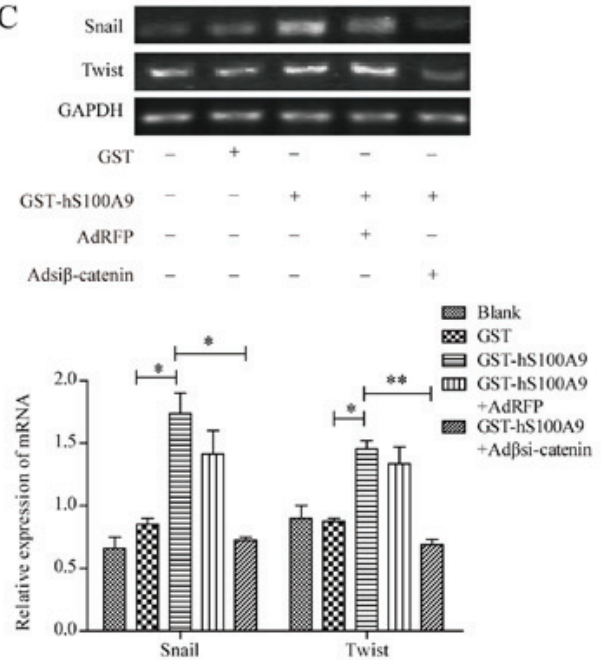

$\mathrm{E}$

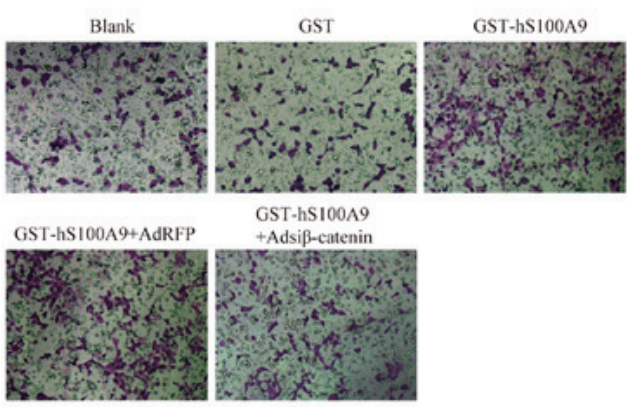

F

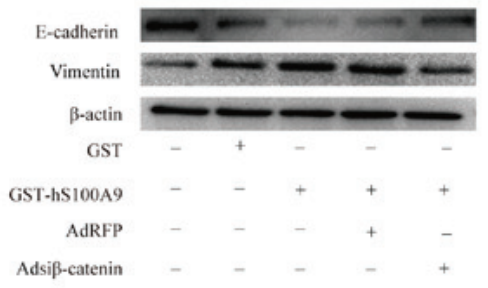

B

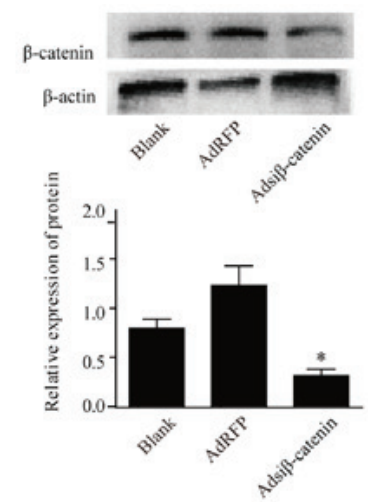

D
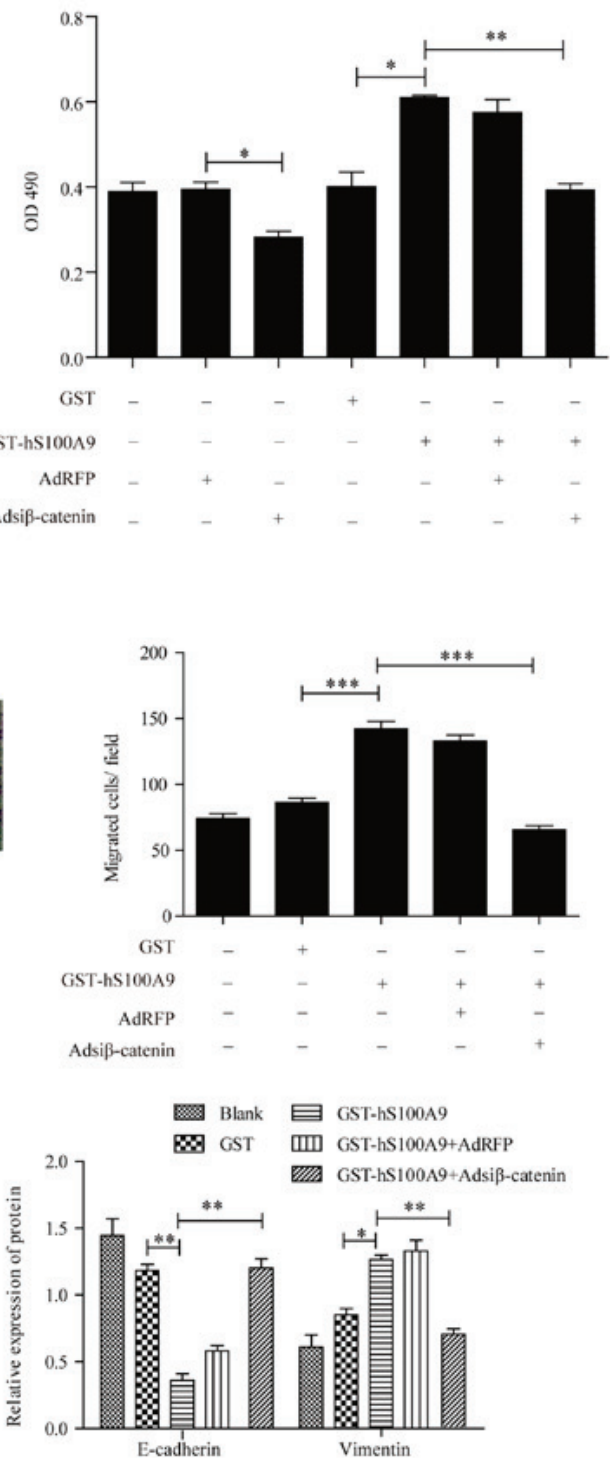

Figure 6. Underlying molecular mechanisms of S100A9-induced promotion of proliferation, migration and EMT in cervical cancer cells. (A) After treatment with or without GST-hS100A9 at different concentrations for $72 \mathrm{~h}$, the proliferation of HeLa cells was measured by an MTT assay. ${ }^{*} \mathrm{P} \leq 0.05$, GST-hS100A9 vs. GST control. (B) Adsi $\beta$-catenin-mediated $\beta$-catenin knockdown in HeLa cells. After infection with Adsi $\beta$-catenin, the expression of $\beta$-catenin was analyzed by western blot analysis. $\beta$-actin was used as internal reference control. ${ }^{*} \mathrm{P} \leq 0.05$, Adsi $\beta$-catenin vs. AdRFP. After infection with Adsi $\beta$-catenin for $24 \mathrm{~h}$, the infected cells were treated with or without GST-hS100A9 at $20 \mu \mathrm{g} / \mathrm{ml}$ for $48 \mathrm{~h}$. The mRNA levels of Snail, and Twist were measured by (C) semi-quantitative polymerase chain reaction and the proliferation of HeLa cells was measured by (D) an MTT assay. GAPDH was used as an internal reference control. ${ }^{*} \mathrm{P} \leq 0.05$, GST vs. GST-hS100A9; ${ }^{* *} \mathrm{P} \leq 0.01$, GST-hS100A9 vs.GST-hS100A9 + Adsi $\beta$-catenin. (E) Transwell migration assay for analyzing the migration of HeLa cells that were infected with Adsi $\beta$-catenin for $24 \mathrm{~h}$ and treated with or without GST-hS100A9 at $20 \mu \mathrm{g} / \mathrm{ml}$ for $48 \mathrm{~h}$. Representative images of transmembrane cells are shown. The mean of transmembrane cells \pm standard deviation per microscopic field of three independent experiments was calculated. Magnification, x100. ${ }^{* * * *} \mathrm{P} \leq 0.001$, GST vs. GST-hS100A9, GST-hS100A9 + AdRFP vs. GST-hS100A9 + Adsi $\beta$-catenin. (F) Western blot for analyzing the expression of EMT markers of HeLa cells that were infected with Adsi $\beta$-catenin for $24 \mathrm{~h}$ and treated with and without GST-hS100A9 at $20 \mu \mathrm{g} / \mathrm{ml}$ for $48 \mathrm{~h}$. $\beta$-actin was used as internal reference control. Data are presented as mean \pm standard deviation of three individual measurements. ${ }^{*} \mathrm{P} \leq 0.05,{ }^{* *} \mathrm{P} \leq 0.01, \mathrm{GST}$ vs. GST-hS100A9, GST-hS100A9 + AdRFP vs. GST-hS100A9 + Adsi 3 -catenin. Ad, adenovirus; Adsi, adenovirus containing small interfering RNA; EMT, epithelial-mesenchymal transition; GST, glutathione S-transferase; OD, optical density; RFP, red fluorescent protein. 
$\mathrm{P} \leq 0.01$; Fig. $6 \mathrm{~F}$ ). These findings demonstrated that S100A9 induced promotion of proliferation, migration and EMT through activation of $\mathrm{Wnt} / \beta$-catenin pathway in cervical cancer cells.

\section{Discussion}

Increasing evidence has indicated that the enhanced motility and invasiveness of cancer cells are associated with the progression of cervical cancer (14-16). In the present study, we observed that overexpression of S100A9 promoted the proliferation and migration of cervical cancer cells, eventually facilitating EMT, which was regulated by the Wnt/ $\beta$-catenin signaling pathway. These findings suggested that S100A9 may be an important carcinogenic factor in the occurrence and progression of cervical cancer and may serve as an attractive therapeutic target for cervical cancer driven by various signaling pathways.

S100 proteins participate in a broad range of intracellular and extracellular functions by regulating calcium balance, cell apoptosis, migration, proliferation, differentiation, energy metabolism and inflammation (17). Overexpressed S100 proteins, including S100A9, were detected in a variety of cancers, including pancreatic, liver, and colorectal carcinomas $(4,13,18)$. Compared with adjacent normal cervical tissues, increased levels of S100A9 were detected in squamous cervical cancer by two-dimensional gel electrophoresis followed by mass spectrometry, and the expression of S100A9 and RAGE in squamous cervical cancer was closely associated with histological differentiation and the tumor progression (6). Furthermore, various studies have shown that S100A9 could promote the proliferation and migration of different cell types, such as fibroblasts (19), MCF-7, MDA-MB-231, SH-EP, Kelly (20), HepG2 (4) and PNT1A cells (21). In accordance with these studies, our results suggested that overexpression of S100A9 enhanced the proliferation and migration properties of HeLa and SiHa cells.

EMT is required for several complex differentiation processes, including embryonic development, wound healing and tumor progression $(22,23)$. Interestingly, Li et al (24) reported that S100A6 could facilitate the metastatic ability and EMT of cervical cancer cells, which was mediated by activating the PI3K/Akt signaling pathway. Additionally, S100A14 was determined to be a mediator of EMT that regulated the proliferation, migration and invasion of human cervical cancer cells (25). Based on these findings, we propose that overexpression of S100A9 resulted in a decrease in E-cadherin and an increase in vimentin expression in cervical cancer cells. Conversely, knockdown of S100A9 exhibited an antagonistic effect on the regulation of E-Cadherin and vimentin. These results suggested that S100A9 could enhance the mesenchymal properties of cervical cancer cells, which may be attributed to the induction of EMT.

The pivotal role of $\mathrm{Wnt} / \beta$-catenin signaling pathway in tumor progression has been generally accepted, and cervical cancer has been linked with the aberrant activation of the Wnt/ $\beta$-catenin pathway $(22,26)$. In the present study, we reported that S100A9 enhanced the accumulation of $\beta$-catenin, and upregulated the mRNA expression of the target genes c-Myc, Snail, and Twist in cervical cancer cells. The results suggested that S100A9 may exert significant effects on the induction of Wnt $/ \beta$-catenin pathway in cervical cancer cells. In addition, previous studies have shown that $\beta$-catenin upregulation was involved in the proliferation and migration of cancer cells $(27,28)$. Our results revealed that $\mathrm{Wnt} / \beta$-catenin pathway was responsible for S100A9-induced promotion cervical cancer cell proliferation and migration, which could be partially suppressed by downregulation of $\beta$-catenin. Furthermore, we observed that activation of the $\mathrm{Wnt} / \beta$-catenin pathway may be responsible for S100A9-induced EMT of cervical cancer cells, which could be partially suppressed by downregulating $\beta$-catenin. These findings suggested that S100A9 could induce EMT in cervical cancer cells via the Wnt/ $\beta$-catenin signaling pathway.

Collectively, the present study revealed that S100A9-induced increases in cervical cancer cell proliferation and migration may be attributed to the promotion of EMT and aberrant activation of the Wnt/ $\beta$-catenin signaling pathway. Additionally, S100A9 may be considered as a novel molecular target for the treatment of cervical cancer in future.

\section{Acknowledgements}

We would like to thank T.C He (Medical Center, The University of Chicago) for the kind provision of the AdGFP, AdRFP, AdS100A9, Adsi $\beta$-catenin recombinant adenoviruses, pGST-moluc and pGST-moluc-hS100A9 plasmids.

\section{Funding}

This work was supported by National Natural Science Foundation grants of China (grant no. 81760475).

\section{Availability of data and materials}

The datasets used and/or analyzed during the present study are available from the corresponding author on request.

\section{Authors' contributions}

$\mathrm{HZ}$ and XL performed most experiments and were major contributors in writing the manuscript. SY, HL, AL, YG, JZ and JX prepared experimental materials and reviewed the article. HS and LD made substantial contributions to the design of the study, and drafted the manuscript and revising it critically for important intellectual content. LZ gave final approval of the version to be published. All authors read and approved the final manuscript.

\section{Ethics approval and consent to participate}

Not applicable.

\section{Patient consent for publication}

Not applicable.

\section{Competing interests}

The authors declare that they have no competing interest. 


\section{References}

1. Siegel RL, Miller KD and Jemal A: Cancer statistics, 2018. CA Cancer J Clin 68: 7-30, 2018.

2. Zhang X, Wang Y, Cao Y, Zhang X and Zhao H: Increased CCL19 expression is associated with progression in cervical cancer. Oncotarget 8: 73817-73825, 2017.

3. Wu R, Duan L, Ye L, Wang H, Yang X, Zhang Y, Chen X, Zhang Y, Weng Y, Luo J, et al: S100A9 promotes the proliferation and invasion of HepG2 hepatocellular carcinoma cells via the activation of the MAPK signaling pathway. Int J Oncol 42 : 1001-1010, 2013.

4. Wu R, Duan L, Cui F, Cao J, Xiang Y, Tang Y and Zhou L: S100A9 promotes human hepatocellular carcinoma cell growth and invasion through RAGE-mediated ERK1/2 and p38 MAPK pathways. Exp Cell Res 334: 228-238, 2015.

5. Zhao Q, He Y, Wang XL, Zhang YX and Wu YM: Differentially expressed proteins among normal cervix, cervical intraepithelial neoplasia and cervical squamous cell carcinoma. Clin Trans Oncol 17: 620-631, 2015.

6. Zhu X, Jin L, Zou S, Shen Q, Jiang W, Lin W and Zhu X: Immunohistochemical expression of RAGE and its ligand (S100A9) in cervical lesions. Cell Biochem Biophys 66: 843-850, 2013.

7. Qureshi R, Arora H and Rizvi MA: EMT in cervical cancer: Its role in tumour progression and response to therapy. Cancer Lett 356B: B321-B331, 2015.

8. Yin C, Li H, Zhang B, Liu Y, Lu G, Lu S, Sun L, Qi Y, Li X and Chen W: RAGE-binding S100A8/A9 promotes the migration and invasion of human breast cancer cells through actin polymerization and epithelial-mesenchymal transition. Breast Cancer Res Treat 142: 297-309, 2013

9. Yang M, Wang M,Li X, Xie Y, Xia X, Tian J,Zhang K and Tang A: Wnt signaling in cervical cancer? J Cancer 9: 1277-1286, 2018.

10. Sun $X$ and Liu Y: Activation of the Wnt $/ \beta$-catenin signaling pathway may contribute to cervical cancer pathogenesis via upregulation of Twist. Oncol Lett 14: 4841-4844, 2017.

11. Shin S, Im HJ, Kwon YJ, Ye DJ, Baek HS, Kim D, Choi HK and Chun YJ: Human steroid sulfatase induces Wnt/ $\beta$-catenin signaling and epithelial-mesenchymal transition by upregulating Twist1 and HIF-1 $\alpha$ in human prostate and cervical cancer cells. Oncotarget 8: 61604-61617, 2017.

12. Busch EL, McGraw KA and Sandler RS: The potential for markers of epithelial-mesenchymal transition to improve colorectal cancer outcomes: A systematic review. Cancer Epidemiol Biomarkers Prev 23: 1164-1175, 2014.

13. Duan L, Wu R, Ye L, Wang H, Yang X, Zhang Y, Chen X, Zuo G, Zhang Y, Weng Y, et al: S100A8 and S100A9 are associated with colorectal carcinoma progression and contribute to colorectal carcinoma cell survival and migration via Wnt/ $\beta$-catenin pathway. PLoS One 8: e62092, 2013.

14. Li X, Zhou Q, Tao L and Yu C: MicroRNA-106a promotes cell migration and invasion by targeting tissue inhibitor of matrix metalloproteinase 2 in cervical cancer. Oncol Rep 38: 1774-1782, 2017.
15. Ye H, Zhang Y, Geng L and Li Z: Cdc42 expression in cervical cancer and its effects on cervical tumor invasion and migration. Int J Oncol 46: 757-763, 2015

16. Wang $\mathrm{C}, \mathrm{Gu}$ W, Zhang Y, Ji Y, Wen Y and Xu X: Nicotine promotes cervical carcinoma cell line HeLa migration and invasion by activating PI3k/Akt/NF- $\kappa$ B pathway in vitro. Exp Toxicol Pathol 69: 402-407, 2017.

17. Xia C, Braunstein Z, Toomey AC, Zhong J and Rao X: S100 proteins as animportant regulator of macrophage inflammation. Front Immunol 8: 1908, 2018.

18. Chen KT, Kim PD, Jones KA, Devarajan K, Patel BB, Hoffman JP, Ehya H, Huang M, Watson JC, Tokar JL, et al: Potential prognostic biomarkers of pancreatic cancer. Pancreas 43: 22-27, 2014

19. Shibata F, Ito A, Ohkuma Y and Mitsui K: Mitogenic activity of S100A9 (MRP-14). Biol Pharm Bull 28: 2312-2314, 2005.

20. Ghavami S, Rashedi I, Dattilo BM, Eshraghi M, Chazin WJ, Hashemi M, Wesselborg S, Kerkhoff C and Los M: S100A8/A9 at low concentration promotes tumor cell growth via RAGE ligation and MAP kinase-dependent pathway. J Leukoc Biol 83: 1484-1492, 2008

21. Hermani A, De Servi B, Medunjanin S, Tessier PA and Mayer D: S100A8 and S100A9 activate MAP kinase and NF-kappaB signaling pathways and trigger translocation of RAGE in human prostate cancer cells. Exp Cell Res 312: 184-197, 2006.

22. Jolly MK, Ware KE, Gilja S, Somarelli JA and Levine H: EMT and MET: Necessary or permissive for metastasis? Mol Oncol 11: 755-769, 2017.

23. Klymkowsky MW and Savagner P: Epithelial-mesenchymal transition: A cancer researcher's conceptual friend and foe. Am J Pathol 174: 1588-1593, 2009.

24. Li A, Gu Y, Li X, Sun H, Zha H, Xie J, Zhao J, Huang M, Chen L, Peng Q, et al: S100A6 promotes the proliferation and migration of cervical cancer cells via the PI3K/Akt signaling pathway. Oncol Lett 15: 5685-5693, 2018.

25. Wang X, Yang J, Qian J, Liu Z, Chen H and Cui Z: S100A14, a mediator of epithelial-mesenchymal transition, regulates proliferation, migration and invasion of human cervical cancer cells. Am J Cancer Res 5: 1484-1495, 2015.

26. Cain JM, Ngan H, Garland S and Wright T; FIGO Working Group on Combating Cervical Cancer: Control of cervical cancer: Women's options and rights. Int J Gynaecol Obstet 106: 141-143, 2009.

27. Lustig B and Behrens J: The Wnt signaling pathway and its role in tumor development. J Cancer Res Clin Oncol 129: 199-221, 2003.

28. Kahn M: Can we safely target the WNT pathway? Nat Rev Drug Discov 13: 513-532, 2014

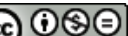

This work is licensed under a Creative Commons Attribution-NonCommercial-NoDerivatives 4.0 International (CC BY-NC-ND 4.0) License. 